\title{
EDITORIAL
}

\section{Management of spontaneous pneumothorax: back to the future}

\author{
A.C. Miller
}

Most practising thoracic physicians and surgeons trained in the days when it was accepted that, in all but the most minor cases, patients with spontaneous pneumothorax required removal of intrapleural air as soon as possible, the standard method being intercostal drainage using a trocar and cannula. Such tubes were commonly inserted by inexperienced doctors, who were often unable to give adequate analgesia to the parietal pleura. It was common for drains to be poorly positioned, accurate placement was difficult to maintain, and, even if these problems were avoided, patients would be in hospital for several days with limited mobility and frequently troublesome chest pain. For many patients, the experience was so unpleasant that the possibility of a repeat episode made the idea of prophylactic thoracic surgery welcome. It is hard to comprehend how management deteriorated to such a state of affairs from previous clinical practice.

Only 50 yrs ago, in the days before effective chemotherapy, "collapse therapy" was frequently performed as first-line treatment for pulmonary tuberculosis. In order to collapse a pulmonary cavity, the amount of air introduced into the chest was often considerably more than that which it was, subsequently, felt essential to remove in spontaneous pneumothorax; in some patients, this was performed bilaterally at the same session. These were out-patient procedures; in spite of the amount of air in the pleural cavity most patients resumed normal lives and occupation until their next "refill clinic", which was necessary because the intrapleural air reabsorbed spontaneously. The technique involved the use of a needle of considerably smaller bore than modern intercostal tubes, without local anaesthesia.

How did these earlier chest specialists manage their patients with spontaneous pneumothorax? One study describes a unit's experience of 119 consecutive patients from 1954-1963 [1], and is typical of medical practice at the time; $74 \%$ of patients (33 of the whole cohort had underlying chronic lung disease) had no intervention at all, were not admitted to hospital, and were usually encouraged to remain at work. In half of the patients requiring drainage, this was performed by simple aspiration; indeed, to achieve this, some groups used the same equipment which in refill clinics was being used to induce pneumothorax.

Of course, there are two important differences between therapeutic and spontaneous pneumothorax. In the latter, the lung may collapse completely, and also there is an underlying defect in the lung which may not seal.

Mayday University Hospital, Croydon, Surrey CR7 7YE, UK.
However, in most cases, neither of these possibilities justifies the automatic insertion of a large-bore intercostal drain.

Five principles must be remembered. Firstly, the presence of intrapleural air is not in itself an indication for intervention. It should be recognized that, whereas difficulty in reinflating the lung is a common problem in malignant pleural effusions, this is not the case in spontaneous pneumothorax, where failure is almost always due to persistent air leak.

Secondly, management of the pneumothorax should depend primarily upon its clinical effect rather than its radiographic size. Young patients, in particular, providing pain is controlled, can tolerate substantial collapse without drainage, a plan that could be disastrous in a patient with chronic symptomatic underlying lung disease, even with a small pneumothorax.

Thirdly, the presence of major lung collapse does not necessarily imply a continuing air leak; there is a trend for such an association, but in the majority of such patients simple aspiration leads to rapid re-expansion of the lung (see below). This seems surprising; perhaps sealing of the leak is easier when lung tissue is compressed.

Fourthly, the concept of tension pneumothorax should be reserved for patients with pneumothorax due to barotrauma. Such a situation in a patient on positive pressure ventilation, which can rapidly threaten life, can only occur in spontaneous pneumothorax if there is a so-called "ballvalve effect", with air leaking into the pleural cavity in each inspiration but unable to equilibrate with that in the atmosphere. But, how often does this actually happen? Many practitioners assume that the radiographic appearance of depression of the ipsilateral diaphragm in association with mediastinal shift contralaterally is due to such a mechanism; in fact, since simple aspiration is often successful in such cases, this must be a false conclusion. When such radiographic changes are noted, a decision to intervene should still be based primarily on the patient's clinical state.

Finally, when drainage is required, there is no longer any justification for tube drainage in the first instance. The historic practice of simple aspiration, which is extremely easy to learn and carry out, which involves minimal discomfort for the patient, and which does not demand hospitalization, should always be attempted first. Since 1982, at least seven studies in 233 patients from the United States [2-3], Australia [4], United Kingdom [5-7] and Singapore [8] have shown that where simple aspiration is attempted, satisfactory re-expansion is achieved in $70 \%$ of patients with normal lung function; even in those 
with underlying chronic lung disease the figure is $35 \%$. Even with $>50 \%$ lung collapse, simple aspiration is successful in $62 \%$. These results are comparable to intercostal tube drainage and a clinical trial [6] found that the subsequent recurrence rate is similar whichever drainage method is adopted; this is hardly surprising, since the objective of both methods is merely to drain the pleural cavity. There are three possible scenarios: 1) the hole has sealed and aspiration will suffice; 2) the hole remains widely patent, so that several days of intercostal drainage are necessary before there is much chance of resolution; or 3) there is a small air leak, so that after apparently successful aspiration the lung slowly deflates. The only situation in which an intercostal drain should be considered as the first drainage procedure is in those patients with severe underlying lung disease, in whom surgery is thought to be hazardous; if successful (as is the case in a third of such patients), the presence of the tube offers an opportunity for chemical pleurodesis.

It was widespread ignorance and misunderstanding of these principles that led the British Thoracic Society (representing both respiratory physicians and thoracic surgeons) to publish guidelines [9] for the initial management of spontaneous pneumothorax, giving advice about: when observation alone is sufficient; which patients require admission; when and how to perform simple aspiration and tube drainage; and when to seek expert advice (in the UK, most such patients are initially seen by nonspecialists). Subsequent widespread adoption of this approach, which represented a significant departure in clinical practice for many of those who agreed to its introduction, has substantially reduced the number of admissions and unnecessary interventions, and has proved popular with those patients who had previous unpleasant experience of unnecessary intercostal tube drains.

This latter period coincides with the increasing adoption of video-assisted thoracoscopic surgery for suitable patients, and the paper by SCHRAMEL et al. [10] confirms the experience of other groups that this has a valuable role in the management of spontaneous pneumothorax. Indeed, the advantages of this technique in reducing hospital stay and postoperative pain make it an attractive option, which is perhaps why the authors operated on all patients with spontaneous pneumothorax. How should we re-evaluate the indications for surgical intervention?

It is indisputable that surgery should be considered when there is a persistent air leak. In those with "normal" lungs, surgery is necessary when leakage continues for a week, whereas in those with significant underlying lung disease a longer period of conservative management may be successful [11]. However, most procedures are performed to prevent recurrence, and there are few situations, for example a patient who is likely to be spending some time remote from good medical care, where prophylactic surgery can be considered after the first episode. But in general, after how many episodes should surgery be advised? Twenty two percent of the historical control group of the Amsterdam study recurred within $2 \mathrm{yrs}$, and only another 5\% subsequently, in line with other studies [12]; therefore, it appears that $73 \%$ of their later patients had unnecessary operations, a possibility that they concede. Even though the risk of further episodes increases with the number that the patient has already had, a substantial proportion of those who have had more than two will have no more. On the one hand, the advantages of the newer techniques should make surgery more attractive to patients. On the other hand, a return to the more conservative approach of former years, with many now observed as out-patients and some others having simple aspiration without hospital admission, is already making patients less enthusiastic about operation after just two episodes.

\section{References}

1. Stradling P, Poole G. Conservative management of spontaneous pneumothorax. Thorax 1966; 21: 145-149.

2. Bevelaqua FA, Aranda C. Management of spontaneous pneumothorax with small-lumen catheter manual aspiration. Chest 1982; 81: 693-694.

3. Delius RE, Obeid FN, Horst H, Sorensen VJ, Fath JJ, Bivins BA. Catheter aspiration for simple pneumothorax. Arch Surg 1989; 124: 833-836.

4. Markos J, McGonigle P, Phillips MJ. Pneumothorax: treatment by small-lumen catheter aspiration. Aust $N Z$ J Med 1990; 20: 775-781.

5. Archer GJ, Hamilton AAD, Upadhyay R, Finlay M, Grace PM. Results of simple aspiration of pneumothoraces. Br J Dis Chest 1985; 79: 177-182.

6. Harvey J, Prescott RJ. Simple aspiration versus intercostal tube drainage for spontaneous pneumothorax in patients with normal lungs. BMJ 1994; 309: 1338-1339.

7. Seaton D, Yoganathan K, Coady T, Barker R. Spontaneous pneumothorax: marker gas technique for predicting outcome of manual aspiration. BMJ 1991; 302: 262265.

8. $\mathrm{Ng} \mathrm{AWK}$, Chan KW, Lee SK. Simple aspiration of pneumothorax. Singapore Med J 1994; 35: 50-52.

9. Miller AC, Harvey JT. Guidelines for the management of spontaneous pneumothorax. BMJ 1993; 307: 114116.

10. Schramel FMNH, Sujedja TG, Braber JCE, van Mourik JC, Postmus PE. Cost-effectiveness of video-assisted thorascopic surgery versus conservative treatment for first time or recurrent spontaneous pneumothorax. Eur Respir $J$ 1996; 9: 1821-1825.

11. Mathur R, Cullen J, Kinnear WJM, Johnston IDA. Time course of persistent air leak in spontaneous pneumothorax. Respir Med 1995; 89: 129-132.

12. Lippert HL, Lund O, Blegvad S, Larsen HV. Independent risk factors for cumulative recurrence rate after first spontaneous pneumothorax. Eur Respir J 1991; 4: 324-331. 